The Egyptian Journal of Hospital Medicine (October 2020) Vol. 81 (5), Page 1993-2000

\title{
Serum Golgi Protein 73 as a Biomarker for Liver Fibrosis in Chronic Hepatitis C Patients
}

\author{
Hala A Abdel-Azeez ${ }^{1}$, Samar M Sharaf ${ }^{1}$, Eman A Elshamy ${ }^{2}$, Abeer A Fikry ${ }^{1}$ \\ Departments of ${ }^{1}$ Clinical Pathology and ${ }^{2}$ Tropical Medicine, Faculty of Medicine, Zagazig University \\ *Corresponding author: Abeer A Fikry, Mobile: 00201029153181, Email: abfikry@ gmail.com
}

\begin{abstract}
Background: Serum Golgi protein 73 (GP73) is a promising biomarker of liver fibrosis, but most data are from hepatitis B virus-related liver diseases rather than hepatitis C virus (HCV).

Objective: To evaluate the use of GP73 as a marker for liver fibrosis in chronic HCV patients and to correlate it with fibrosis staging of liver biopsy and other laboratory findings.

Subjects and Methods: The study included 37 chronic HCV patients who were subdivided according to liver biopsy results into chronic HCV patients without fibrosis (10) and chronic HCV patients with fibrosis (27). 37 healthy individuals were taken as control. All participants were subjected to determination of liver function tests with calculation of AST-to-Platelet Ratio Index (APRI) and serum GP73 by ELISA.

Results: GP73 was significantly increased in chronic HCV patients with fibrosis when compared to patients without fibrosis and healthy control. A significant positive correlation was observed between GP73 and advanced liver function tests, increased fibrosis stage and APRI in chronic $\mathrm{HCV}$ patients with fibrosis. Based on receiver operating characteristic (ROC) curve analysis, GP73 had an area under curve (AUC) of 0.909 with $81.8 \%$ sensitivity and $93.3 \%$ specificity for prediction of significant fibrosis. On combination with APRI, the sensitivity was increased to $90.9 \%$.

Conclusion: GP73 can be used as serum marker for prediction of significant liver fibrosis in chronic HCV patients either alone or in combination with APRI. It can be also useful in monitoring fibrosis progression.
\end{abstract}

Keywords: Hepatitis C, Golgi protein 73, APRI, Liver fibrosis.

\section{INTRODUCTION}

Hepatitis $\mathrm{C}$ virus (HCV) represents a significant global public health burden, and long-term infection can lead to many serious liver-related outcomes including liver cirrhosis, hepatocellular carcinoma and liver-related mortality ${ }^{(1)}$. The event with the greatest impact on the natural history of chronic $\mathrm{HCV}$ is the progressive formation and accumulation of liver fibrosis. This accumulation of fibrillar extracellular matrix components is the hallmark of the natural history of the disease and significantly influences prognosis ${ }^{(2)}$. Liver fibrosis may progress to cirrhosis and eventually death. However, increasing evidence suggests that even advanced fibrosis is reversible, although end-stage cirrhosis is irreversible and affected patients can only survive with a liver transplant. Estimating the current degree of fibrosis is crucial for determining whether the fibrosis could be reversed with treatment ${ }^{(3)}$.

Liver biopsy is an invasive method that has long been regarded as the 'gold standard' for staging liver fibrosis. However, several issues prevent the routine use of liver biopsy as a clinical tool, including risk of injury to the patient, variable accessibility of the damaged section of the liver, high cost, sampling errors and inaccuracy due to inter- and intra-observer variability of pathologic interpretations (4). Instead, several noninvasive methods for fibrosis assessment have been proposed as the alternatives to liver biopsy, such as fibrosis index based on four factors (FIB-4), the ASTto-

platelet ratio index (APRI), and transient elastography, which are based on blood indices and imaging modalities, respectively ${ }^{(5)}$. Non-invasive assessment of liver fibrosis is a focus of continuing interest to find a marker that is cost-effective, easy to perform with standard laboratory tests, accurately reflective of the stage of hepatic fibrosis and valid over a wide patient population ${ }^{(6)}$.

In normal livers, Golgi protein 73 (GP73) is a 73 $\mathrm{kDa}$ transmembrane glycoprotein constitutively expressed by biliary epithelial cells, but only minimally by hepatocytes. Hepatocellular expression of GP73 mRNA and protein is dramatically upregulated in acute or chronic hepatitis, regardless of the etiology, and accompanies the advanced fibrogenesis stage. With the recovery of acute hepatitis, GP73 expression reverses, whereas biliary epithelial cell expression remains unchanged. GP73 overexpression also occurs in activated hepatic stellate cells, which are the major feature of liver fibrosis. GP73 has been considered a useful indicator in evaluating liver impairment or fibrosis ${ }^{(7)}$. 
Previous researches about GP73 were mainly focused on hepatitis B virus (HBV) related liver diseases and others reported it as a potential diagnostic marker for hepatocellular carcinoma, the diagnostic potential of serum GP73 in chronic HCV-related liver diseases remains to be investigated. Our study aimed to evaluate the use of GP73 as a marker for liver fibrosis in chronic hepatitis $\mathrm{C}$ patients and to correlate it with fibrosis staging of liver biopsy and other laboratory findings.

\section{SUBJECTS AND METHODS}

This is a case-control study, which was carried out on 37 patients suffering from chronic hepatitis $\mathrm{C}$ virus, and 37 age and sex matched healthy individuals, taken as control. Patients were recruited from the Inpatient Sections and Outpatient Clinics of Tropical Medicine Department, Zagazig University Hospitals during the period from March 2019 to August 2019.

Chronic HCV patients were diagnosed by RTPCR and elevated AST, ALT more than 6 months ${ }^{(8)}$. Patients were subdivided according to liver biopsy results into chronic HCV patient without fibrosis (10) and chronic HCV patient with fibrosis (27). Staging of fibrosis was done according to French Metavir Cooperative Study Group ${ }^{\left({ }^{(9)}\right.}$. Significant liver fibrosis was defined as $\geq$ F2. Patients under therapy for HCV, with hepatocellular carcinoma, liver cirrhosis F4 and other systemic diseases were excluded from the study.

\section{Ethical approval:}

Written informed consent was obtained from all individual participants included in the study.

The study was approved by the Local Ethics Committee of Faculty of Medicine, Zagazig University.

\section{Laboratory investigations:}

All participants were subjected to determination of platelets count (Sysmex XS 5001), prothrombin concentration (CA 1500 Sysmex), liver function tests (Cobas 8000, Roch Diagnostics) and calculation of AST-to-Platelet Ratio Index (APRI) ${ }^{(\mathbf{1 0})}$.

A cut-off value $>0.5$ was previously established for APRI for diagnosis of significant fibrosis (11). Détermination of serum GP73 was performed by Enzyme-Linked Immunosorbent Assay using Uscn life ELISA kit (Wuhan USCN Business Co).

\section{Statistical analysis}

Statistical analyses were performed by SPSS 22.0 software (International Business Machines Corporation, New York, USA). Continuous variables were expressed as mean \pm standard deviation (SD) or median and interquartile range (IQR). Categorical variables were expressed as percentages. The differences of groups were analyzed by One-Way ANOVA, or Kruskal-Wallis (KW) rank sum test according to the data's distribution.

Post-hoc Fisher's Least Significant Difference test (LSD) or Tamhane's T tests were used according to homogenesity of variances. In addition, the chi-square test $(\chi 2)$ was applied to compare the rates of the classification data. Pearson and Spearman's rank correlation coefficient tests were used to describe the association between two variables.

The diagnostic effectiveness of variables was assessed by the area under the receiver operating characteristic (AUROC) curve with 95\% confidence interval (CI), was used to identify optimal cut-off values with maximum sensitivity and specificity. All tests of significance were two-tailed, and $\mathrm{P} \leq 0.05$ was considered statistically significant. 


\section{RESULTS}

Demographic data and laboratory findings of the studied groups are presented in table (1).

Table (1): Demographic data and laboratory findings of the studied groups

\begin{tabular}{|c|c|c|c|c|}
\hline $\begin{array}{l}\text { Demographic data } \\
\& \text { \& } \\
\text { Laboratory finding }\end{array}$ & $\begin{array}{c}\text { Healthy control } \\
(\mathbf{n}=\mathbf{3 7})\end{array}$ & $\begin{array}{c}\text { Chronic HCV } \\
\text { without fibrosis }(n=10)\end{array}$ & $\begin{array}{c}\text { Chronic HCV } \\
\text { with fibrosis }(n=27)\end{array}$ & p-value \\
\hline $\begin{array}{l}\text { Age (years) } \\
\text { Median } \\
\text { Range }\end{array}$ & $\begin{array}{c}43 \\
22-55\end{array}$ & $\begin{array}{c}47 \\
39-55\end{array}$ & $\begin{array}{c}44 \\
20-53\end{array}$ & 0.245 \\
\hline \begin{tabular}{|l|} 
Sex\#\# \\
Male \\
Female
\end{tabular} & $\begin{array}{l}18(48.6 \%) \\
19(51.4 \%)\end{array}$ & $\begin{array}{l}7(70 \%) \\
3(30 \%)\end{array}$ & $\begin{array}{l}19(70.4 \%) \\
8(29.6 \%)\end{array}$ & 0.165 \\
\hline Fibrosis stage (F0/F1/F2/F3) & ---- & $10 / 0 / 0 / 0$ & $0 / 5 / 17 / 5$ & --- \\
\hline Platelets count $\left(\mathrm{x}^{3} 0^{3} / \mathrm{mm}^{3}\right)$ & $\begin{array}{c}250 \\
(180-300)\end{array}$ & $\begin{array}{c}252.5 \\
(150-280)\end{array}$ & $\begin{array}{c}180^{1} \\
(107-280)\end{array}$ & $<0.001$ \\
\hline Prothrombin conc. $(\%)$ & $\begin{array}{c}99 \\
(92-105)\end{array}$ & $\begin{array}{c}98 \\
(92-102)\end{array}$ & $\begin{array}{c}93^{2} \\
(65-105)\end{array}$ & 0.037 \\
\hline Total bilirubin (mg/dl) & $\begin{array}{c}0.6 \\
(0.3-0.9)\end{array}$ & $\begin{array}{c}0.8^{1} \\
(0.7-2)\end{array}$ & $\begin{array}{c}1.4^{1} \\
(0.5-4)\end{array}$ & $<0.001$ \\
\hline Direct bilirubin (mg/dl) & $\begin{array}{c}0.2 \\
(0.1-0.2)\end{array}$ & $\begin{array}{c}0.2^{1} \\
(0.1-0.5)\end{array}$ & $\begin{array}{c}0.6^{1} \\
(0.1-1.8)\end{array}$ & $<0.001$ \\
\hline Albumin (g/dl)\# & $4.4 \pm 0.4$ & $4.4 \pm 0.2$ & $3.4 \pm 0.3^{1}$ & $<0.001$ \\
\hline Alkaline phosphatase (U/L) & $\begin{array}{c}160 \\
(100-200)\end{array}$ & $\begin{array}{c}119 \\
(100-190)\end{array}$ & $\begin{array}{c}152 \\
(86-240)\end{array}$ & 0.096 \\
\hline ALT (U/L) & $\begin{array}{c}22 \\
(18-26)\end{array}$ & $\begin{array}{c}37^{1} \\
(28-42)\end{array}$ & $\begin{array}{c}48^{1} \\
(12-117)\end{array}$ & $<0.001$ \\
\hline AST (U/L) & $\begin{array}{c}22 \\
(18-30)\end{array}$ & $\begin{array}{c}37^{1} \\
(32-55)\end{array}$ & $\begin{array}{c}45^{1} \\
(38-105)\end{array}$ & $<0.001$ \\
\hline APRI & $\begin{array}{c}0.2 \\
(0.1-0.3)\end{array}$ & $\begin{array}{c}0.3^{1} \\
(0.2-0.7)\end{array}$ & $\begin{array}{c}0.6^{1} \\
(0.4-2)\end{array}$ & $<0.001$ \\
\hline GP73 (ng/ml) & $\begin{array}{c}4 \\
(1-5)\end{array}$ & $\begin{array}{c}3.9 \\
(2.5-4)\end{array}$ & $\begin{array}{c}5^{1} \\
(3.5-9.5)\end{array}$ & $<0.001$ \\
\hline
\end{tabular}

Data are presented as median (range), mean \pm SD \#, or number (percentage) \#\#

$\mathrm{P} \leq 0.05$ is significant. ${ }^{1}$ significant with other groups ${ }^{2}$ significant with control group

GP73 was significantly increased in chronic HCV with fibrosis compared to HCV without fibrosis and control groups while no significant difference in GP73 between chronic HCV without fibrosis and control group (figure 1). 


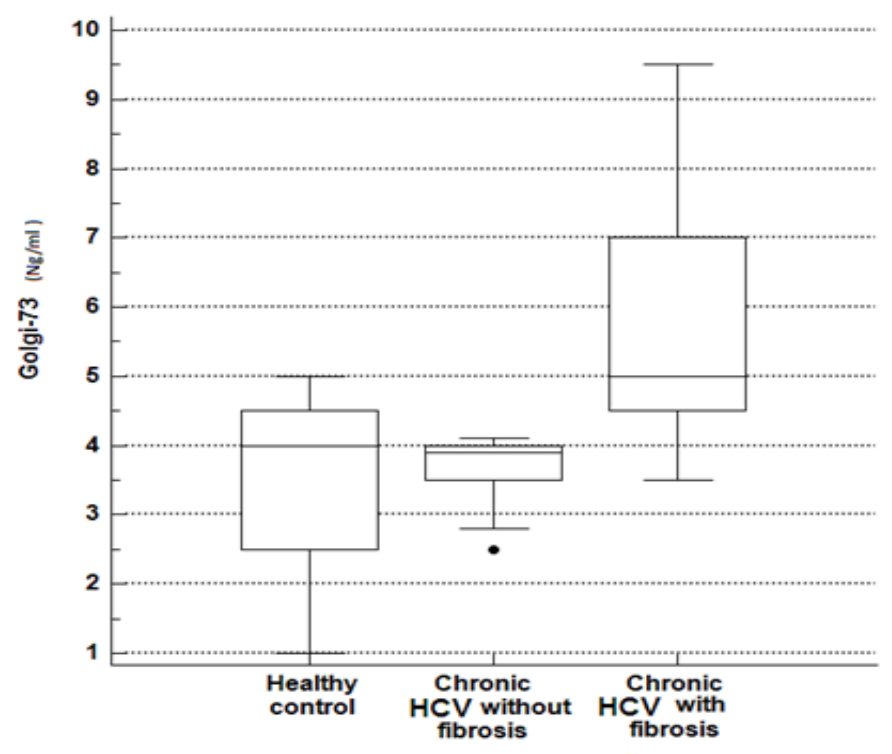

Figure (1): Box-plot of GP73 in the studied groups. Horizontal lines represent median values, boxes represent 25, 75 percentile and error bars represent ranges.

GP73 was significantly positively correlated with total and direct bilirubin in chronic HCV patients without fibrosis. There was a significant negative correlation between GP73 \& platelet count, prothrombin concentration and albumin. While, there was a significant positive correlation between GP73 \& total bilirubin, direct bilirubin, alkaline phosphatase, ALT, AST, fibrosis staging and APRI in chronic HCV patients with fibrosis (table 2, figures 2 \& 3).

Table (2): Correlation between GP73 and selected study parameters

\begin{tabular}{|c|c|c|c|c|}
\hline \multirow[t]{3}{*}{ Laboratory findings } & \multicolumn{4}{|c|}{ GP73 } \\
\hline & \multicolumn{2}{|c|}{ Chronic $\mathrm{HCV}$ without fibrosis $(\mathrm{n}=10)$} & \multicolumn{2}{|c|}{ Chronic HCV with fibrosis $(n=27)$} \\
\hline & $r$ & $\mathrm{p}$ & $\mathrm{r}$ & $p$ \\
\hline $\begin{array}{l}\text { Platelets count } \\
\left(\mathrm{X}^{3} \mathbf{3}^{3} / \mathbf{m m}^{3}\right)\end{array}$ & -0.324 & 0.361 & -0.418 & 0.030 \\
\hline $\begin{array}{l}\text { Prothrombin conc. } \\
(\%)\end{array}$ & -0.282 & 0.430 & -0.477 & 0.012 \\
\hline Total bilirubin (mg/dl) & +0.638 & 0.047 & +0.775 & $<0.001$ \\
\hline $\begin{array}{l}\text { Direct bilirubin } \\
\text { (mg/dl) }\end{array}$ & +0.646 & 0.043 & +0.569 & 0.002 \\
\hline Albumin (g/dl) & -0.466 & 0.174 & -0.540 & 0.004 \\
\hline $\begin{array}{l}\text { Alkaline phosphatase } \\
\text { (U/L) }\end{array}$ & -0.558 & 0.094 & +0.390 & 0.044 \\
\hline ALT (U/L) & -0.205 & 0.569 & +0.795 & $<0.001$ \\
\hline AST (U/L) & +0.105 & 0.772 & +0.715 & $<0.001$ \\
\hline Fibrosis staging & --- & --- & +0.653 & $<0.001$ \\
\hline APRI & +0.284 & 0.427 & +0.680 & $<0.001$ \\
\hline
\end{tabular}

$\mathrm{P} \leq 0.05$ is significant 


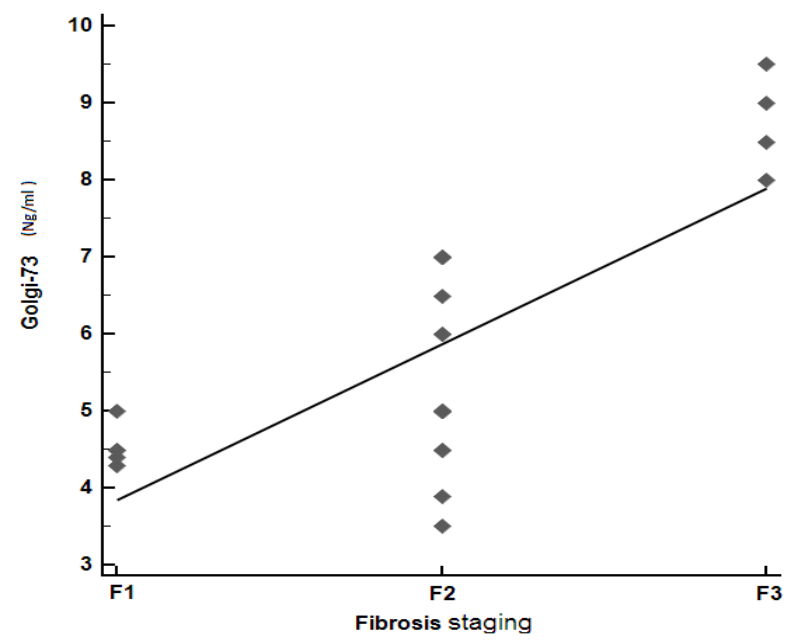

Figure (2): Correlation between fibrosis staging and GP73 in chronic HCV patients with fibrosis

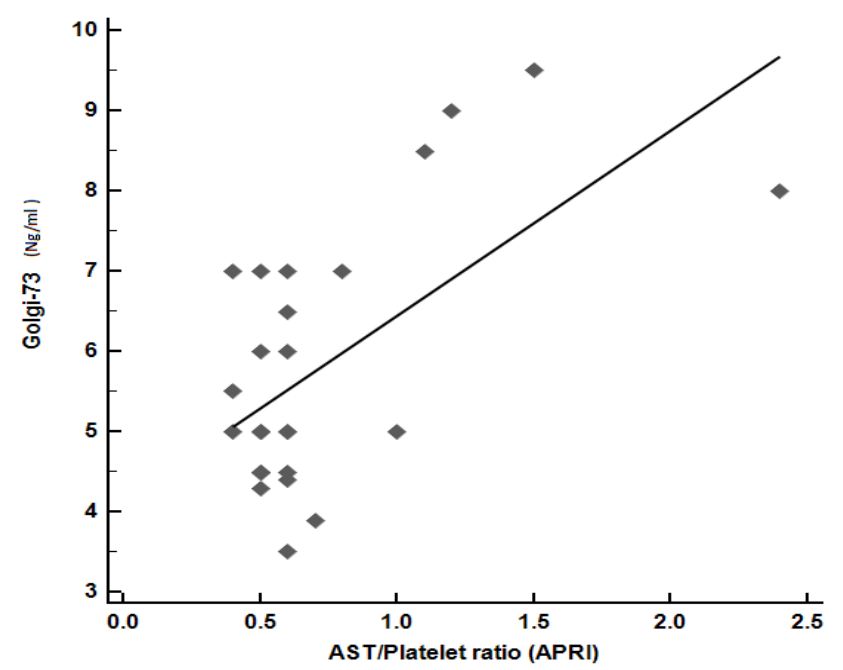

Figure (3): Correlation between APRI and GP73 in chronic HCV patients with fibrosis

ROC curve analysis of GP73 as a predictor of significant fibrosis ( $\geq F 2$ ) in chronic HCV patients revealed an AUC of 0.909. At cut-off value $>4.5 \mathrm{ng} / \mathrm{ml}$ for GP73, 18 out of 22 chronic HCV patients with significant fibroses were correctly diagnosed and 1 out of 15 chronic HCV patients without fibrosis was misclassified as having fibrosis. When GP73 and APRI were used in combination for diagnosis of liver fibrosis, 20 out of 22 were correctly dignosed (figures $4 \& 5$, table 3).

Table (3): GP73 and combined APRI \& GP73 as a predictor for significant fibrosis in chronic hepatitis patients; ROC curve analysis

\begin{tabular}{|l|c|c|c|c|c|c|}
\hline Parameter & $\begin{array}{c}\text { Sensitivity } \\
\%\end{array}$ & $\begin{array}{c}\text { Specificity } \\
\%\end{array}$ & $\begin{array}{c}\text { Positive } \\
\text { Predictive } \\
\text { Value \% } \\
\text { (PPV) }\end{array}$ & $\begin{array}{c}\text { Negative } \\
\text { Predictive Value } \\
\%(\mathbf{N P V})\end{array}$ & Accuracy & $\begin{array}{c}\text { AUC } \\
(\mathbf{9 5 \%} \text { CI) }\end{array}$ \\
\hline GP73 & $81.8 \%$ & $93.3 \%$ & $94.7 \%$ & $77.8 \%$ & $86.5 \%$ & $0.909(0.809-1.009)$ \\
\hline $\begin{array}{l}\text { Combined } \\
\text { APRI and } \\
\text { GP73 }\end{array}$ & $90.9 \%$ & $73.3 \%$ & $83.3 \%$ & $84.6 \%$ & $83.3 \%$ & $0.935(0.854-1.015)$ \\
\hline
\end{tabular}


ROC Curve

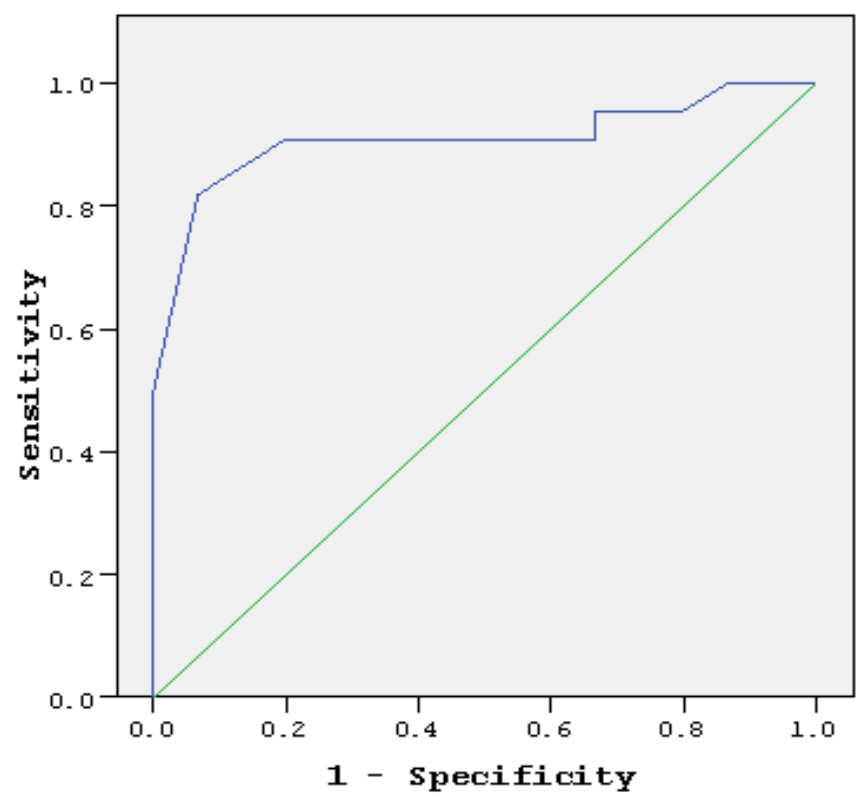

Figure (4): Receiver operating characteristic (ROC) curve of GP73 as a predictor for significant fibrosis in chronic hepatitis patients.

\section{ROC Curve}

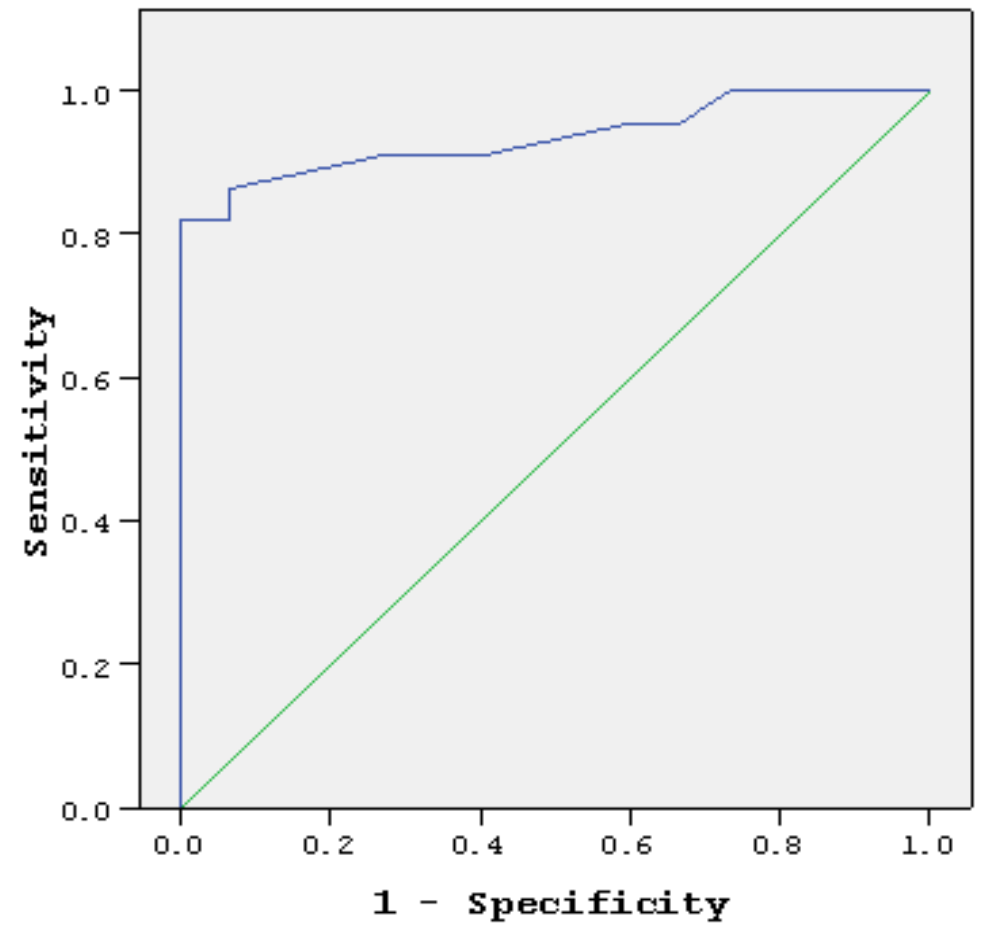

Figure (5): Receiver operating characteristic (ROC) curve of APRI \& GP73 as a predictor for significant fibrosis in chronic HCV patients 


\section{DISCUSSION}

Chronic HCV patients with no or minimal fibrosis at presentation appear to progress slowly and treatment possibly could delay or withheld progression. On the other hand, patients with significant fibrosis (i.e. septal or bridging fibrosis) progress almost invariably are going to cirrhosis over 10 to 20 years period ${ }^{(10)}$. Fortunately, antiviral treatment can reverse even early cirrhosis ${ }^{(\mathbf{1 2}, \mathbf{1 3})}$. So, to better manage the chronic hepatitis $\mathrm{HCV}$ patients, it is critical to evaluate and monitor the grade of inflammation and the stage of liver fibrosis and cirrhosis. Interest in identifying and describing liver fibrosis using serum markers has been on the rise. Serum markers offer a cost effective alternative to liver biopsy for both patients and clinicians. In addition, being less invasive, there is a low risk of sampling error and no observer-related variability. Moreover, measurements may be performed repeatedly over time, allowing for ongoing monitoring of fibrosis ${ }^{(\mathbf{1 4})}$.

Our study showed that GP73 was significantly increased in chronic HCV patients with fibrosis when compared to patients without fibrosis and healthy control. A significant positive correlation was also observed between GP73 and increased fibrosis stage in chronic HCV patients. The relationship between GP73 levels and lesions of HCV-related liver disease was investigated by Qian et al. ${ }^{(15)}$ who found that serum GP73 rose in parallel with the severity of hepatitis, fibrosis and even cirrhosis. Moreover, several studies have demonstrated that GP73 was increased in HBV-related liver diseases. Xu and his colleagues ${ }^{(16)}$ reported an increase in serum GP73 with increasing fibrosis in HBV patients. Also, Liu et al. ${ }^{(17)}$ demonstrated that GP73 was significantly increased in HBV-related liver fibrosis patients compared to healthy controls. Gu et al. ${ }^{(18)}$ reported that GP73 concentrations in patients with liver disease were three-fold higher than in healthy individuals.

In chronic liver diseases, GP73 and mRNA expression increases gradually, not only in the hepatocytes, but also in activated stellate cells ${ }^{(\mathbf{1 9})}$, which is commonly considered the most important cell type involved in hepatic fibrogenesis ${ }^{(20)}$. Therefore maximal GP73 expressions are seen in fully established cirrhosis (19). GP73 over-expression is induced initially by inflammation, specifically by inflammatory cytokines such as IL-6, possibly in response to chronic active viral infection ${ }^{(21)}$.

We observed that serum GP73 was significantly positively correlated with advanced liver function tests in HCV patients with fibrosis. Similar results were reported by Qian et al. ${ }^{(15)}$ in chronic HCV patients and Xu et al. (22) in chronic HBV patients. A significant positive correlation was observed between GP73 and APRI in chronic HCV patients with fibrosis in this study. Liu et al. ${ }^{(17)}$ and $\mathrm{Xu}$ et al. ${ }^{(22)}$ reported significant positive correlation between serum GP73 and other serum markers of liver fibrosis; serum hyaluronic acid, laminin, type III procollagen protein and type IV collagen.

In our study, ROC curve analysis revealed an AUC of 0.909 , at cutoff $>4.5 \mathrm{ng} / \mathrm{ml}$, GP73 had $81.8 \%$ sensitivity, $93.3 \%$ specificity, PPV $94.7 \%$ and NPV 77.8 $\%$ with $86.5 \%$ accuracy in prediction of significant fibrosis in chronic $\mathrm{HCV}$ patients. A comparable results were reported previously in prediction of significant fibrosis in HBV patients by Wei et al. ${ }^{(23)}$. Serum GP73 is considered as a biomarker for liver diseases with clinically proven sensitivity and specificity ${ }^{(24,25)}$. Gu and his colleagues ${ }^{(\mathbf{1 8})}$ used GP73 to detect liver cirrhosis with a sensitivity of $88.4 \%$.

When we combined APRI and serum GP73 as a diagnostic marker for significant liver fibrosis, the ROCAUC increased to 0.935 . Twenty out of 22 chronic HCV patients with significant fibrosis were correctly diagnosed with increased sensitivity (90.9\%) and negative predictive value (84.6\%).

\section{CONCLUSION}

Serum GP73 is significantly increased in chronic $\mathrm{HCV}$ patients with fibrosis and is correlated with fibrosis staging determined by liver biopsy. It can be used as serum marker for prediction of significant liver fibrosis in chronic HCV patients either alone or in combination with APRI. It can be also a useful marker in monitoring fibrosis progression. Further studies on large sample size and in different liver diseases are recommended to confirm these results.

Funding: None.

Conflicts of interest: The authors declare that they had no conflicts of interest.

\section{REFERENCES}

1. Aghemo A, Dore G, Hatzakis A et al. (2015): Estimating HCV disease burden. J Viral Hepat., 3 (4): 1-3.

2. Friedman $S$ (2008): Mechanisms of hepatic fibrogenesis. Gastroenterology, 134: 1655- 1669.

3. Friedman $\mathbf{S}$ (2003): Liver fibrosis from bench to bedside. J. Hepatol., 38 (1):38-53.

4. Rossi E, Adams L, Bulsara M et al. (2007): Assessing liver fibrosis with serum marker models. Clin Biochem Rev., 28 (1): 3-10.

5. Trivedi P, Bruns T, Cheung A et al. (2014): Optimising risk stratification in primary biliary cirrhosis: AST/platelet ratio index predicts outcome independent of ursodeoxycholic acid response. Journal of Hepatology, 60 ( 6): $1249-1258$. 
6. Fabris C, Smirne C, Toniutto P et al. (2008): Usefulness of six non- proprietary indirect markers of liver fibrosis in patients with chronic hepatitis C. Clin Chem Lab Med., 46: 253- 259.

7. Riener M, Stenner F, Liewen H et al. (2009): Golgi phosphoprotein 2 (GOLPH2) expression in liver tumors and its value as a serum marker in hepatocellular carcinomas. Hepatology, 49: 1602-1609.

8. Shiratori Y, Yokosuka R, Ihori M et al. (1999): Prospective study of interferon therapy for compensated cirrhotic patients with chronic hepatitis $\mathrm{C}$ by monitoring serum hepatitis C RNA. Hepatology, 29: 1573-80.

9. French METAVIR Cooperative Study Group (1994): Intraobserver and interobserver variations in liver biopsy interpretation in patients with chronic hepatitis C. Hepatology, 20: 15-20.

10. Wai C, Greenson J, Fontana $R$ et al. (2003): A simple noninvasive index can predict both significant fibrosis and cirrhosis in patients with chronic hepatitis C. Hepatology, 38: 518- 526.

11. Sebastiani G, Halfon P, Castera L et al. (2009): SAFE biopsy: a validated method for large- scale staging of liver fibrosis in chronic hepatitis C. Hepatology, 49: 1821- 1827.

12. Grgurevic I, Bozin T, Madir A (2017): Hepatitis $C$ is now curable, but what happens with cirrhosis and portal hypertension afterwards? Clinical and Experimental Hepatology, 4: 181-186.

13. Sun Y, Zhou J, Wu X et al. (2018) : Quantitative assessment of liver fibrosis (qFibrosis) reveals precise outcomes in Ishak "stable" patients on anti-HBV therapy. Scientific Reports, 8 (1): 2989-93.

14. Zhou K, Lu L (2009): Assessment of fibrosis in chronic liver diseases. Journal of Digestive Diseases, 10 (1): 7-14.

15. Qian X, Zheng S, Wang L et al. (2019): Exploring the diagnostic potential of serum golgi protein 73 for hepatic necroinflammation and fibrosis in chronic HCV Infection with different stages of liver injuries. Dis Markers, 3: 1-10.

16. Xu Z, Shen J, Pan X et al. (2018): Predictive value of serum Golgi protein 73 for prominent hepatic necroinflammation in chronic HBV infection. Journal of Medical Virology, 90 (6): 1053-1062.

17. Liu X, Wan X, Li Z et al. (2011): Golgi protein 73 (GP73), a useful serum marker in liver diseases. Clin Chem Lab Med., 49: 1311-1316.

18. Gu Y, Chen W, Zhao Y et al. (2009) : Quantitative analysis of elevated serum Golgi protein-73 expression in patients with liver diseases. Ann Clin Biochem., 46: 38-43.

19. Iftikhar R, Kladney $R$, Havlioglu $N$ et al. (2004): Disease- and cell-specific expression of GP73 in human liver disease. Am J Gastroenterol., 99: 1087-1095.

20. Carpino G, Franchitto A, Morini $S$ et al. (2004): Activated hepatic stellate cells in liver cirrhosis. A morphologic and morphometrical study. Ital $\mathbf{J}$ Anat Embryol., 109: 225-238.

21. Liang H, Block T, Wang $M$ et al. (2012): Interleukin-6 and oncostatin $\mathrm{M}$ are elevated in liver disease in conjunction with candidate hepatocellular carcinoma biomarker GP73. Cancer Biomark., 11: 161-171.

22. Xu Z, Liu L, Pan X et al. (2015): Serum Golgi protein 73 (GP73) is a diagnostic and prognostic marker of chronic HBV liver disease. Medicine, 94: 659-63.

23. Wei H, Li B, Zhang R et al.(2013): Serum GP73, a marker for evaluating progression in patients with chronic HBV infections. PLoS One, 8 (2): 53862-67.

24. Marrero J, Romano P, Nikolaeva $O$ et al. (2005): GP73, a resident Golgi glycoprotein, is a novel serum marker for hepatocellular carcinoma. J Hepatol., 43: 1007-1012.

25. Mao Y, Yang H, Xu H et al. (2010): Golgi protein 73 (GOLPH2) is a valuable serum marker for hepatocellular carcinoma. Gut, 59: 1687-1693. 